\title{
Fungal community composition and function after long-term exposure of northern forests to elevated atmospheric $\mathrm{CO}_{2}$ and tropospheric $\mathrm{O}_{3}$
}

\author{
Ivan P. Edwards ${ }^{1^{*}}$ and Donald R. Zak ${ }^{1,2}$
}

\section{Supplementary Material}

1. Species accumulation curves $(\bullet)$, and the calculated ICE ( $\bullet$ ) and Chao 1 (triangle) estimates of fungal diversity under aspen and aspen birch at FACE II. Top panel; Ascomycetes. Bottom panel; Basidiomycetes

2. Neighbor Joining tree showing the phylogenetic relationship between Pezizomycotina operational taxonomic units and GenBank derived reference sequences. Bootstrap support $>75 \%$ is indicated above nodes, and strongly supported branches $(>=90 \%)$ are indicated with bold type.

3. Maximum Likelihood Consensus tree of the Pezizomycotina from the FACE II site. Bootstrap support $>65 \%$ is indicated above nodes, and strongly supported branches $(>=90 \%)$ are indicated with bold type.

4. Neighbor Joining tree showing the phylogenetic relationship between Basidiomycotina operational taxonomic units and GenBank derived reference sequences. Bootstrap support $>75 \%$ is indicated above nodes, and strongly supported branches $(>=90 \%)$ are indicated with bold type.

5. Maximum Likelihood Consensus tree of the Basidiomycotina from the FACE II site. Bootstrap support $>65 \%$ is indicated above nodes, and strongly supported branches ( $>=90 \%$ ) are indicated with bold type. 
Suppl. Fig. 1
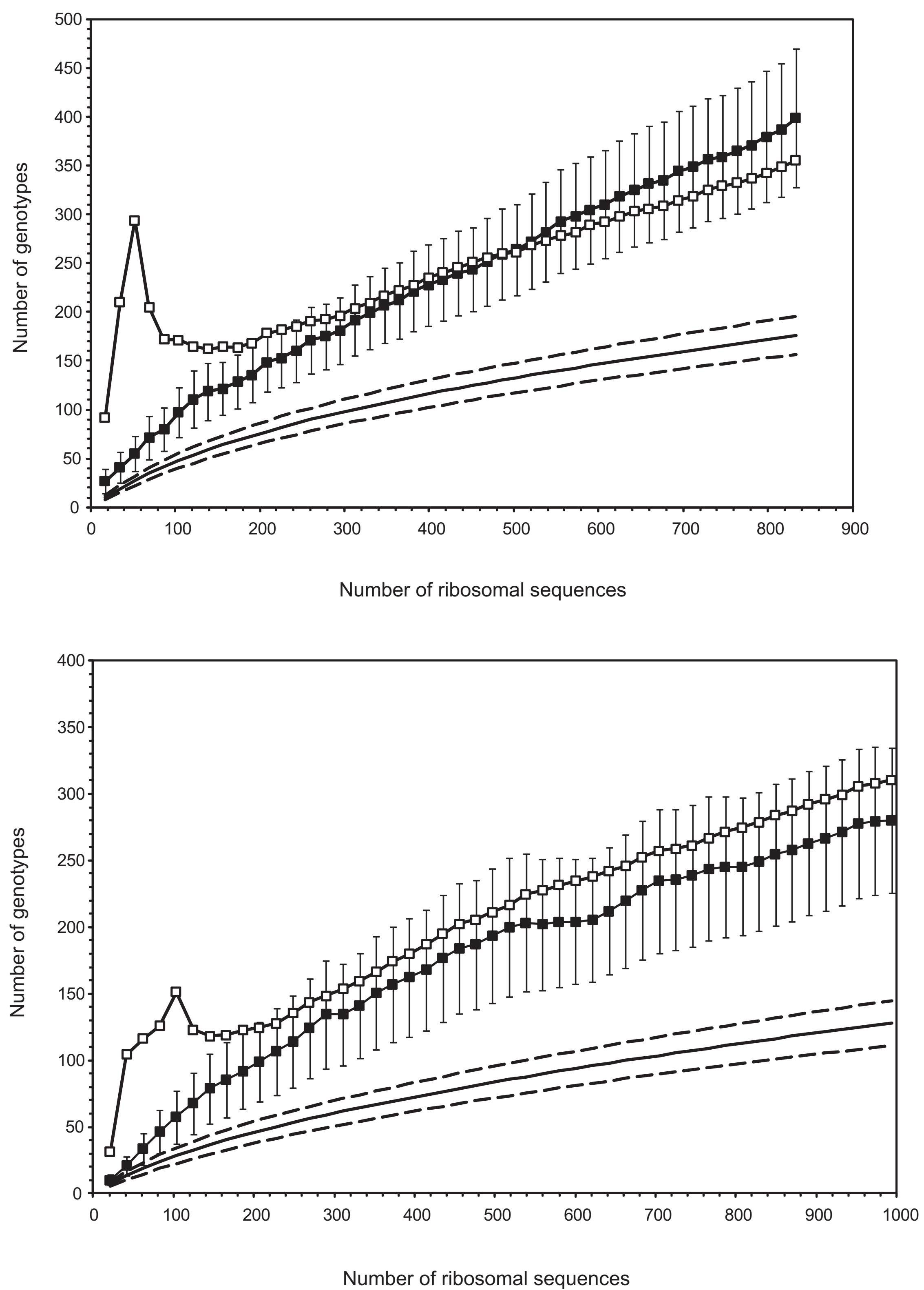
EF596821 Phialea strobilina

- asc07203

$94-a s c 07099$
$\square$

asc07057

99 EU035444 Protoventuria alpina

asc07188

GU727552 Cistella acuum

$\mathbf{H}_{\text {asc } 07058}$

- asco7001 2 Y442323 Cryptosporiops is ericae

- asc07104 asc07115 07085

$\mathrm{L}_{\text {asc07029 }}^{\operatorname{asc} 07137}$

asc07161

- asc07030
asc07110

AF335454 Bisporella citrina

- asc07027

87 asc07071 asc07107

- $\begin{array}{r}\text { asc07028 } \\ \text { asc07126 }\end{array}$

- asc07183

asc07093 as 07092

asc07108

[ asc07108

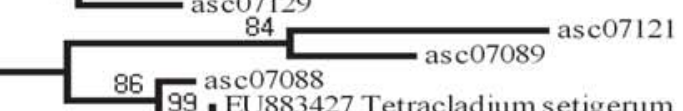$$
4 \text { asc07031 }
$$

_ as as 0706

Г asc07037

- as 007062

- asc07073

$\sqrt{\text { - asc } 07148}$ asc07060

rE asc07148

7 r asc07061

a $\mathrm{c} 07034$
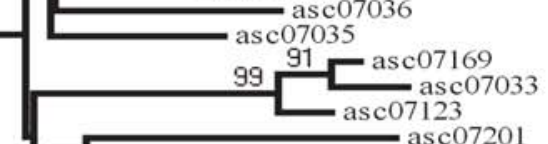

- asc07063 asc0720

asc07101 DQ470981 Microglossum rufum

L- asc07127

LAB517942 Geomyces pannorum

GQ 154618 Phaeomoniella effusa
asc07118 07095 99 A Y660917 Aspergillus fumigatus

AF454167 Aspergillus nidulans

FJ904676 Cryptodiscus microstomus

99 - asc07135

FJ904685 Cryptodiscus rhopaloides

Eurotiomycetes

Aspergillus (Eurotiomycetes)

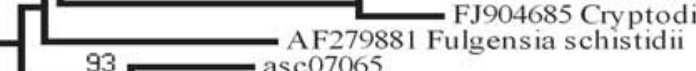

$93-$ asc07065

asc07182

$99-2$ asc07100

asc 07040

Asbolus crenulatus

$99 \square$ asc0706

Pezizomycetes II

$\begin{array}{ll}93 & \text { DQ470948 Peziza vesiculosa } \\ \text { asc07068 }\end{array}$

$9 6 \longdiv { \mathrm { asc } 0 7 1 5 5 }$

is asc07117

DQ384574 Peziza badia

Pezizomycetes III

94

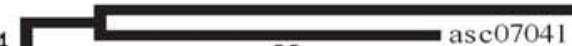

99 07003

(1)

89 A Y544664 Gy romitra esculenta

A Y544673 Gy romitra californica

99 AB278159 Candida zey lanoides 


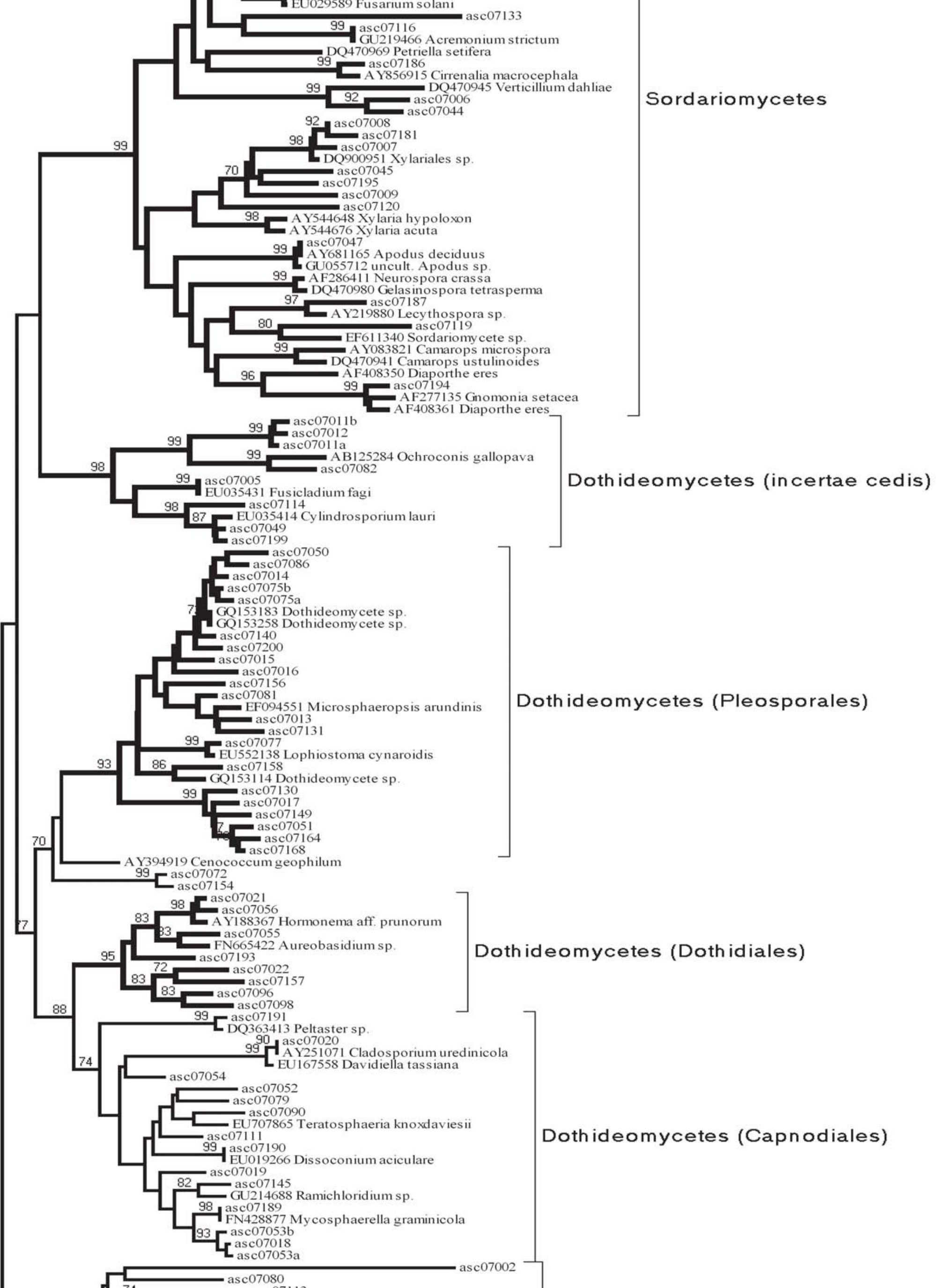




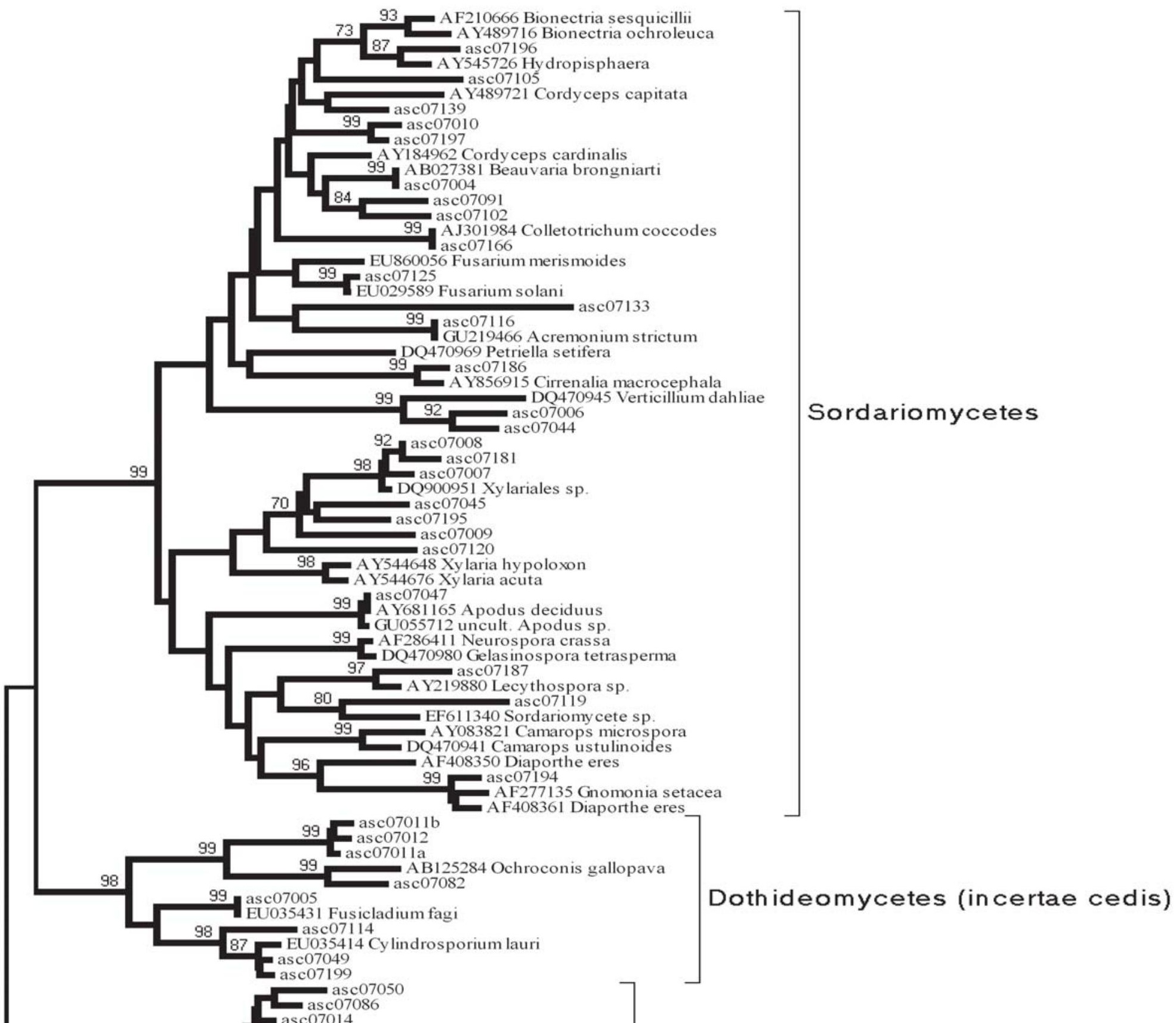




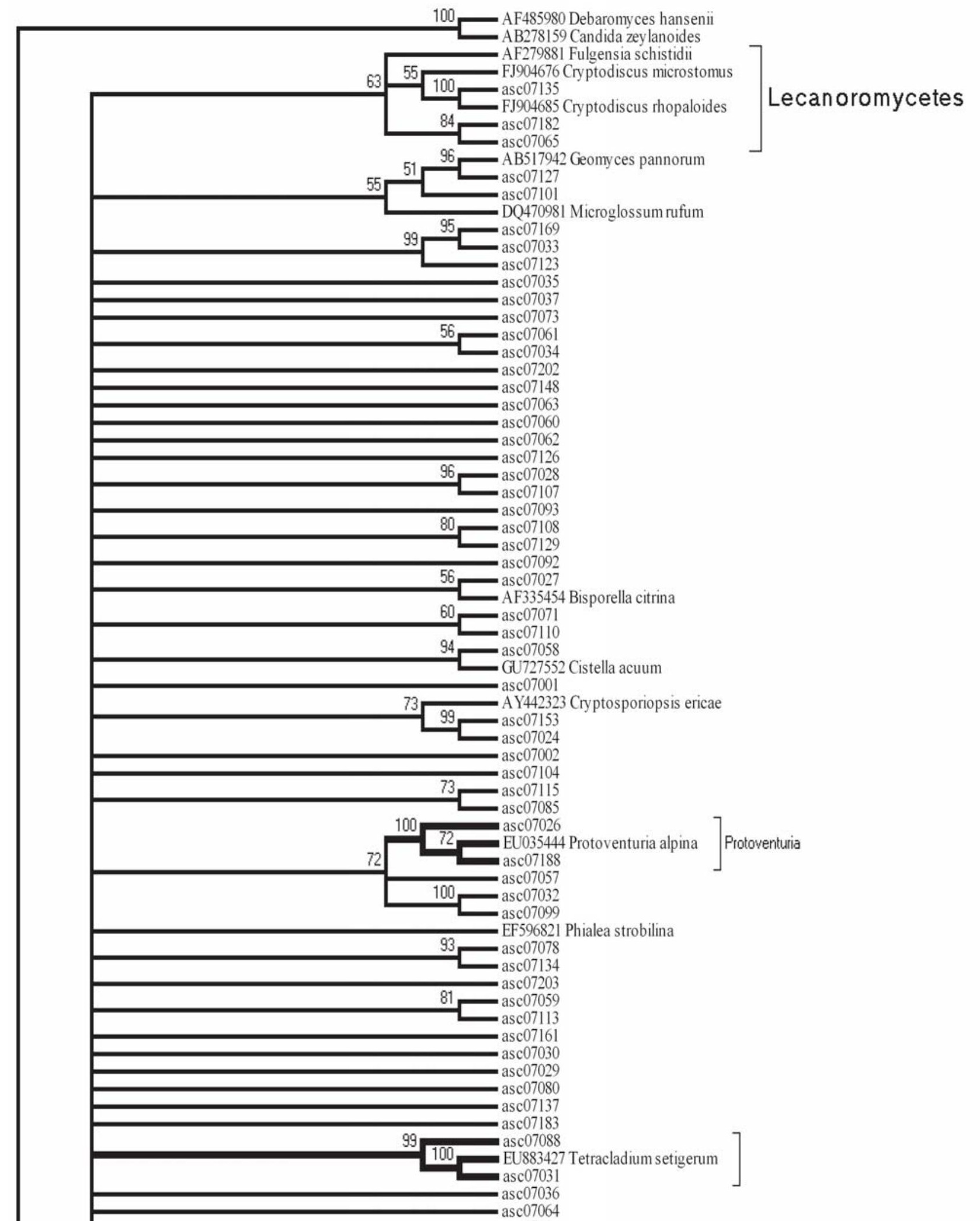




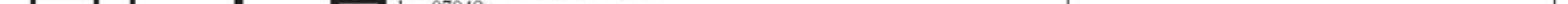




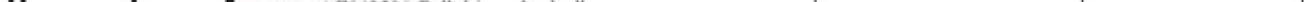




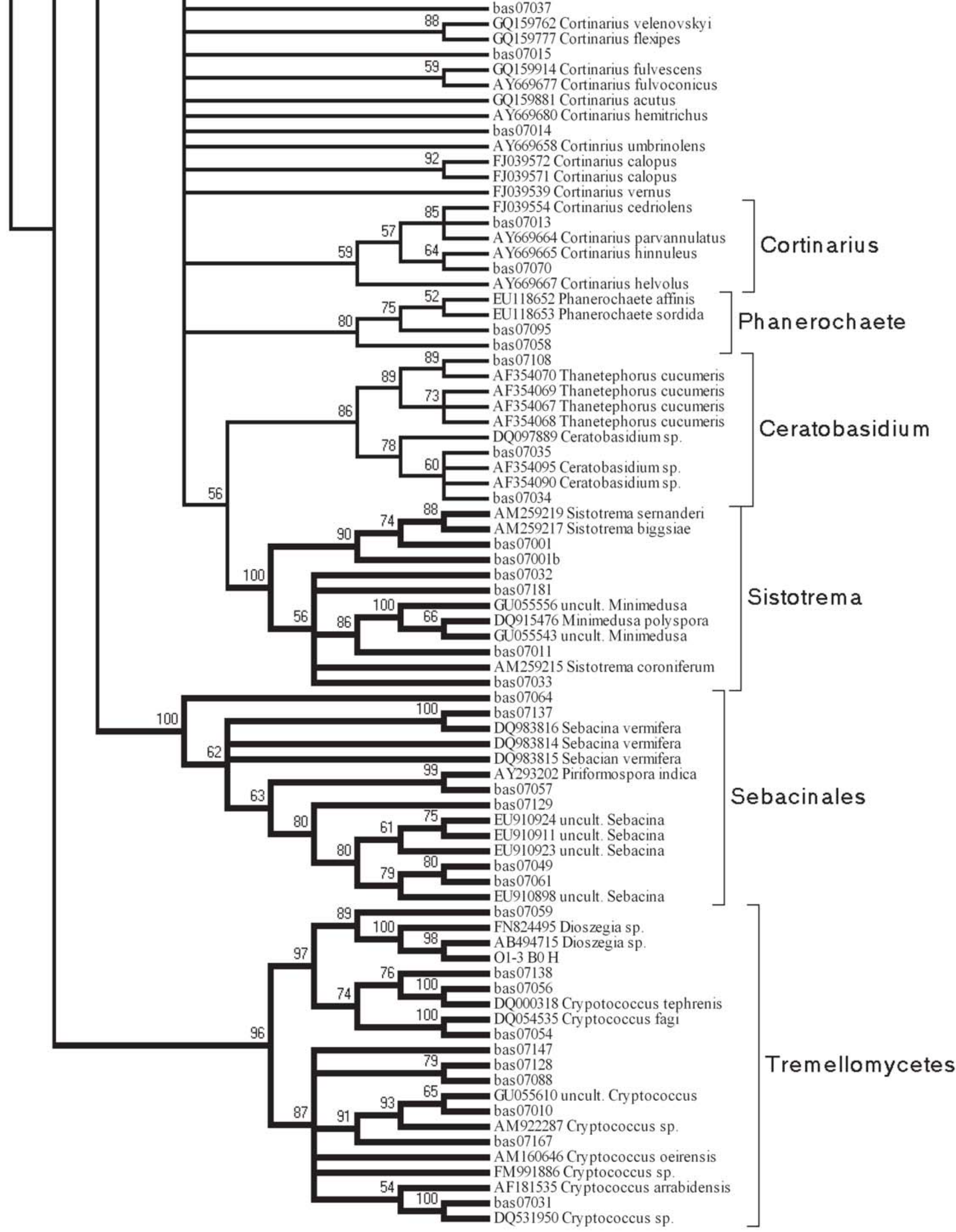

Suppl. Fig. 5. 


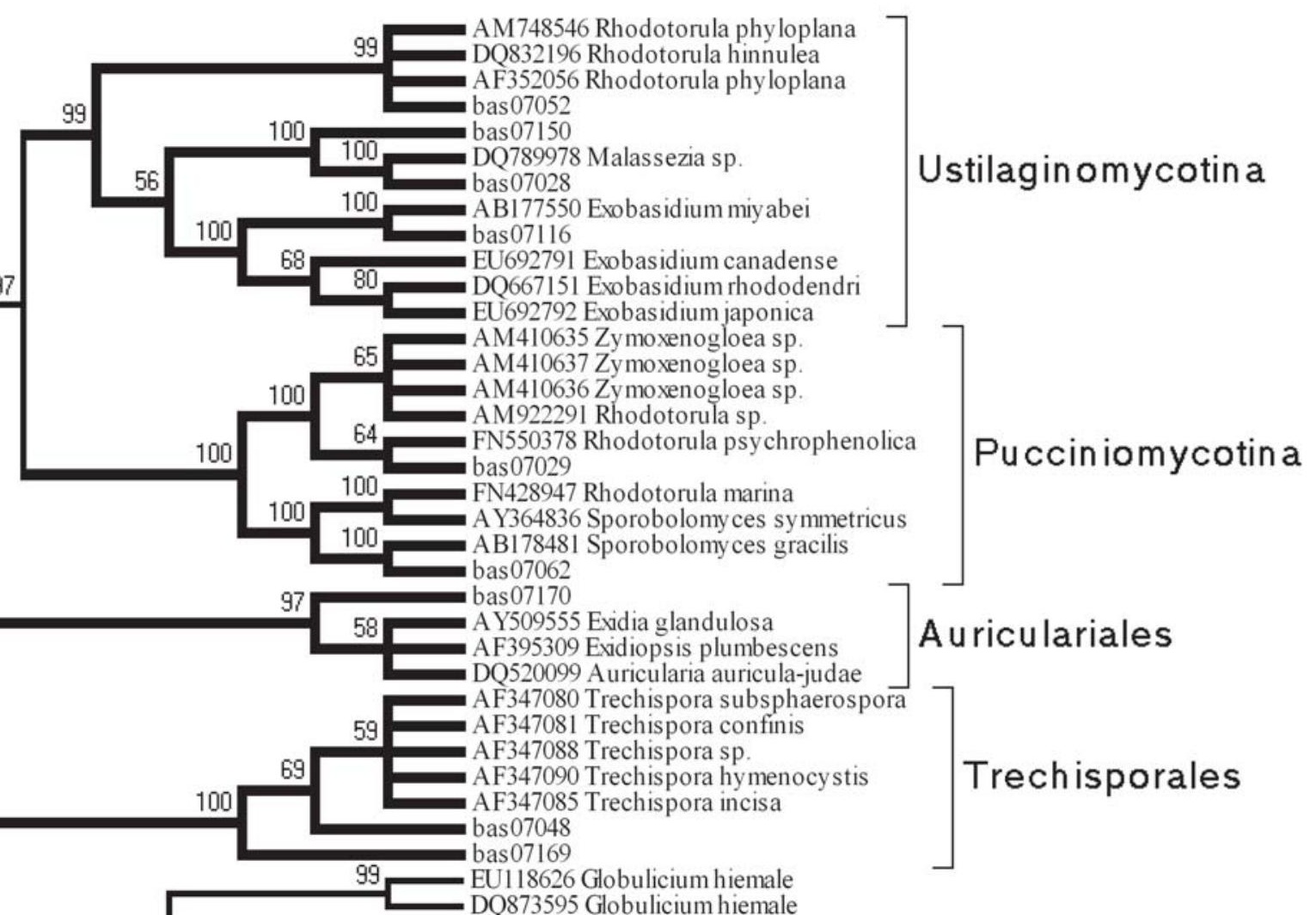



